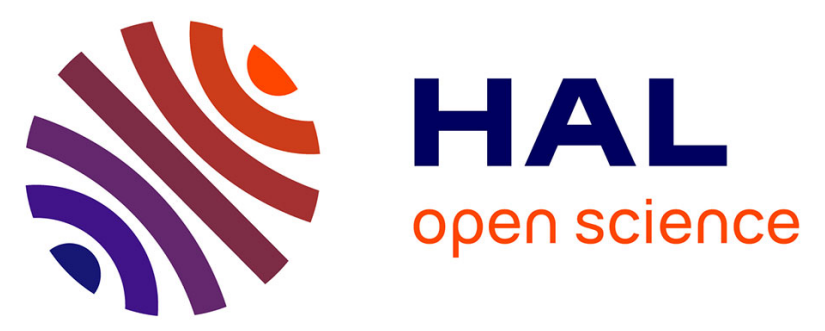

\title{
Données complémentaires sur les anciennes exploitations ferrifères du district de Belle-Isle-en-Terre. Place des gîtes du Nord-Ouest de la France dans les extractions nationales vers 1840
}

Louis Chauris

\section{To cite this version:}

Louis Chauris. Données complémentaires sur les anciennes exploitations ferrifères du district de BelleIsle-en-Terre. Place des gîtes du Nord-Ouest de la France dans les extractions nationales vers 1840. Annales de Bretagne et des Pays de l'Ouest, 1992, 99 (2), pp.165-168. 10.3406/abpo.1992.3425 . insu-01860962

\section{HAL Id: insu-01860962 \\ https://hal-insu.archives-ouvertes.fr/insu-01860962}

Submitted on 3 Feb 2021

HAL is a multi-disciplinary open access archive for the deposit and dissemination of scientific research documents, whether they are published or not. The documents may come from teaching and research institutions in France or abroad, or from public or private research centers.
L'archive ouverte pluridisciplinaire HAL, est destinée au dépôt et à la diffusion de documents scientifiques de niveau recherche, publiés ou non, émanant des établissements d'enseignement et de recherche français ou étrangers, des laboratoires publics ou privés. 


\section{Données complémentaires sur les anciennes exploitations ferrifères} du district de Belle-Isle-en-Terre. Place des gîtes du Nord-Ouest de la France dans les extractions nationales vers 1840

\section{Louis Chauris}

\section{Abstract}

Quantitative data allow to state precisely the place of the Belle-Isle-en-Terre and north-western France iron deposits with regard to the French iron workings before the industrial revolution.

\section{Résumé}

Des données chiffrées permettent de préciser la place des minières du district de Belle-Isle-en-Terre et du Nord-Ouest de la France par rapport aux extractions ferrifères françaises à la veille de la révolution industrielle.

\section{Citer ce document / Cite this document :}

Chauris Louis. Données complémentaires sur les anciennes exploitations ferrifères du district de Belle-Isle-en-Terre. Place des gîtes du Nord-Ouest de la France dans les extractions nationales vers 1840. In: Annales de Bretagne et des pays de l'Ouest. Tome 99, numéro 2, 1992. pp. 165-168;

doi : https://doi.org/10.3406/abpo.1992.3425

https://www.persee.fr/doc/abpo_0399-0826_1992_num_99_2_3425

Fichier pdf généré le 04/04/2018 


\title{
Données complémentaires sur les anciennes exploitations ferrifères du district de Belle-Isle-en-Terre. Place des gîtes du nord-ouest de la France dans les extractions nationales vers 1840
}

\author{
par Louis Chauris
}

Des données chiffrées permettent de préciser la place des minières du district de Belle-Isle-en-Terre et du Nord-Ouest de la France par rapport aux extractions ferrifères françaises à la veille de la révolution industrielle.

Quantitative data allow to state precisely the place of the Belle-Isle-en-Terre and north-western France iron deposits with regard to the French iron workings before the industrial revolution.

L'article paru récemment sur les anciennes extractions des minerais de fer dans la région de Belle-Isle-en-Terre en Bretagne Occidentale' peut être utilement complété par des données chiffrées, éparses dans le volumineux traité - à présent presqu'oublié - de Flachat, Barrault et Petiet, publié en $1846^{2}$. Ces statistiques se rapportent essentiellement à l'année 1841 (éventuellement aux deux années suivantes). Le " deuxième groupe » des auteurs qui concerne les « mines et minières du Nord-Ouest de la France ", réunit les départements des Côtes-du-Nord, Morbihan, Ille-et-Vilaine, Loire-Inférieure, Manche, Mayenne, Orne, Sarthe, Eure et Eure-et-Loire ${ }^{3}$.

Le but de ces lignes est de montrer, à l'aide de quelques exemples quantitatifs, le grand intérêt que ce livre monumental méconnu offre pour l'historien soucieux des réalités économiques. La documentation foumie par cet ouvrage 
permet en effet de mieux saisir la place du département des Côtes-du-Nord (et du district de Belle-Isle-en-Terre) dans le cadre de l'Ouest de la France et de l'ensemble du pays ; d'un point de vue plus général, sa lecture facilite la compréhension des problèmes économiques liés à l'industrie du fer en Bretagne et dans les "Pays de l'Ouest », vers 1840, avant le développement de la sidérurgie moderne.

Le département des Côtes-du-Nord offrait alors 13 minières en activité. On note l'absence de concession de mines sensu stricto. Dans l'ensemble du " groupe du Nord-Ouest " qui comprenait alors 103 minières en activité (dont 33 en Bretagne), il n'y avait en 1841 qu'une seule mine (dans l'Orne) ; toute exploitation avait cessé dans le Finistère à la fin de la petite extraction de Landévennec, en 1840. Les gisements cités dans les Côtes-du-Nord étaient groupés respectivement à proximité du fourneau des Salles (minières de Gouarec, produisant 382 tonnes) ${ }^{4}$; des fourneaux du Pas et de Vau-Blanc (minières de Bas-Vallon l'Hermitage, fournissant $268 \mathrm{t}$. de minerai) ; du fourneau de Coat-an-Noz (minières de Lescalon en Plougonver et de Louargat [district de Belle-Isle-enTerre], produisant ensemble 2757 t.).

Les minières de Lescalon ${ }^{\circ}$ exploitaient un hydroxyde de fer (limonite) en veines et amas, soit à ciel ouvert, soit par petits puits. La production était estimée à 1580 tonnes. Le prix élevé de l'extraction $(4,89 \mathrm{f} / \mathrm{t})$ était compensé par l'excellent rendement en minerai propre à la fusion (près de $94 \%)^{7}$. Le bas prix de revient du lavage du minerai brut de Lescalon doit également être souligné $(1,42 \mathrm{f} / \mathrm{t})$, nettement inférieur à la moyenne $(2,54 \mathrm{f})$ (statistique portant sur une trentaine de gites français). A Lescalon, le traitement du minerai brut après extraction consistait en un simple débourbage.

Le " groupe du Nord-Ouest " occupait le $7^{c}$ rang sur 12 pour le nombre des usines ; le $6^{\mathrm{c}}$ rang pour la production de la fonte et le $7^{\mathrm{C}}$ pour la production du fer. Les données économiques relatives aux seuls départements bretons sont regroupées dans le tableau :

\begin{tabular}{|c|c|c|c|c|}
\hline & Usines & Fonte( $\left(^{*}\right)$ & $\operatorname{Fer}\left({ }^{*}\right)$ & $\begin{array}{l}\text { Remarques } \\
\text { (* production en tonnes) }\end{array}$ \\
\hline Côtes-du-Nord & 6 & 2712 & 656 & $\begin{array}{l}\text { Dont le célèbre } \\
\text { établissement des Salles }\end{array}$ \\
\hline Finistère & 1 & & 347 & $\begin{array}{l}\text { Forge de la Villeuneuve } \\
\text { a Brest }\end{array}$ \\
\hline Ille-et-Vilaine & 6 & 3602 & 1010 & $\begin{array}{l}\text { Grand renom de la forge } \\
\text { de Paimpont }\end{array}$ \\
\hline $\begin{array}{l}\text { Loire-Inférieure } \\
\text { Morbihan }\end{array}$ & $\begin{array}{l}4 \\
4\end{array}$ & $\begin{array}{l}1484 \\
2163\end{array}$ & 3669 & $\begin{array}{l}\text { Dont l'usine de Basse-Indre } \\
\text { Position désavantageuse du } \\
\text { département }\end{array}$ \\
\hline Total & 21 & 9961 & 5682 & \\
\hline $\begin{array}{l}\text { Ensemble du gr. } \\
\text { du Nord-Ouest } \\
\% \text { des départ. bretons }\end{array}$ & $\begin{array}{c}68 \\
30,9\end{array}$ & $\begin{array}{c}27839 \\
35,8\end{array}$ & $\begin{array}{c}13979 \\
40,6\end{array}$ & \\
\hline
\end{tabular}


L'importance relativement faible du " groupe du Nord-Ouest " dans les extractions de minerais de fer en France en 1841 - malgré la présence de gites « assez nombreux " - est mise en relation avec le manque de combustible ${ }^{8}$ et la pénurie des moyens de communication. Par rapport à l'ensemble des extractions nationales ( 12 groupes sont ici considérés), le " deuxième groupe " occupe seulement la $7^{\mathrm{e}}$ place pour le poids de minerai extrait (99433 t. sur 2322839 t., soit $4,28 \%$ ) et également la $7^{\circ}$ place pour le poids de " minerai propre à la fusion » (76607 t. sur 1043921 t., soit 7,34 \%). La qualité du minerai brut extrait dans le " groupe du Nord-Ouest " est nettement supérieure à celle de la moyenne nationale : les pourcentages des minerais propres à la fusion par rapport aux minerais bruts sont respectivement de $77 \%$ et de $\mathbf{4 4 , 9 \%}$. Par contre, les frais d'extraction apparaissent singulièrement élevés : 4,79 f. par tonne de minerai extrait ( $4^{e}$ place) ; 6,21 f. par tonne de minerai propre à la fusion $\left(5^{c}\right.$ place) ; les moyennes nationales étant respectivement de $1,82 \mathrm{f}$. et $4,05 \mathrm{f}$.

Plus grave encore est le retard pris par le " groupe du Nord-Ouest 》 de la France dans l'évolution économique générale : ...» c'est un de ceux [sur les 12 groupes] qui a le moins participé au mouvement décidé de progrès qui s'est manifesté dans la plupart des autres groupes »" Si le handicap lié au « dénuement des voies de communication " n'est pas levé et si des perfectionnements ne sont pas adoptés dans le traitement des minerais, les auteurs du traité prévoient que les usines succomberont « devant la concurrence des autres groupes ». Cette prévision allait se réaliser bientôt pour les établissements de Bretagne Occidentale...?

Le petit district de Belle-Isle-en-Terre n'offrait, en définitive, qu'un poids économique très faible au sein de l'industrie ferrifère française vers les années 1840. Il fournissait moins de $3 \%$ de la production du minerai du " groupe du Nord-Ouest " et seulement un peu plus de $1 / 1000^{\circ}$ de la production nationale. En l'absence d'extraction dans le Finistère, en 1841 , le principal intérêt des minières de Plougonver et de Louargat qui alimentaient le haut fourneau de Coatan-Noz, reposait sur la proximité (relative !) de Brest, port de guerre. La production des installations de Coat-an-Noz était acheminée au grand port du Ponant par mer, après avoir gagné Lannion ${ }^{10}$. L'absence de haut fourneau à cette époque dans le Finistère - pourtant bien pourvu de minerai de fer - soulève un problème qui mériterait d'être examiné...". Le microcentre ferrifère constitué par le district de Belle-Isle-en-Terre devait être balayé par la révolution industrielle' ${ }^{2}$. Et l'arrêt du haut fourneau de Coat-an-Noz, dès 1843 - malgré l'effort entrepris, peu avant, de remplacer une partie du charbon de bois par du coke - apparait comme le prélude à la fermeture des installations bretonnes au charbon de bois...

Louis Chauris Université de Brest 


\section{NOTES}

1. L. Chauris et J. Garreau. Les anciennes exploitations ferrifères des environs de BelleIsle-en-Terre. Ann. de Bretagne et des Pays de l'Ouest, t. 97, 2, 1990, p. 133-146.

2. Traité de la fabrication de la fonte et du fer envisagée sous les trois rapports chimique, mćcanique et commercial. Paris, librairie scientifique - industriclle de L. MathIAs, 1846. Cet ouvrage fournit de précieuses statistiques sur l'industric du fer en France avant la révolution industriclle. Il n'est pas cité dans l'imposante liste de références établie par ANDRIEUX, BrULE et Herbaut-Rousseau : Pour une histoire minière et métallurgique de l'Ouest armoricain. Essai de bibliographie critique régionalc. Mém. Soc. Hist. et Archéol. Bretagne, t. LXVI, 1989, p. 437-489. Nous remercions Ph. GENTILHOMME, géologue au BRGM, à qui nous devons la connaissance de ce livre.

3. Le minerai de fer exploité dans le Finistère, en 1840, près de Landévennec, était expédié pour traitement dans le Morbihan. E. DE FourCy, Carte géologique du Finistère, Edit. de Fain et Thunot, Paris, 1844, 196 p. En 1840, les fouilles de Landévennec produisirent 736 tonnes de minerai au prix de revient de 3 f./tonne ; l'exploitation fut bientôt abandonnée par suite des frais élevés de transport du minerai à l'usine de traitement.

4. Tous les chiffres cités dans l'article concernent des données annuclles.

5. Ces chiffres sont sensiblement différents de ceux foumis par E. DE FoURCY (Carte géologique des Côtes-du-Nord, Edit. de Fain et Thunot, Paris, 1844, 172 p.). Cette divergence pourrait être due aux variations dans les productions annuelles (1842, in DE FOURCY) et/ou au fait que les données du traité et de DE FOURCY ne se rapporteraient pas aux mêmes stades d'élaboration du minerai ? Ainsi selon DE FOURCY, la production des minières de Goarec (sic) s'était ćlevéc à 1090 t. de minerai brut ; par contre, la production des minières des environs de Coat-an-Noz n'était plus que de 1400 t. DE FOURCY cite également les minières du Menez (732 t.) et de Catenoy (536 t.), non répertoriées dans le traité.

6. Le Scalon des cartes actuelles. Situé à proximité du lieu-dit Kervataon'.

7. A titre de comparaison, les frais d'extraction (par tonne) des minières de Nomey ot Charmont, dans le Doubs, s'abaissaient à 1,57 f., mais avec un rendement de minerai propre à la fusion de $7 \%$ seulement ! Dans la minière de Montsangcon, Haute-Marne, les frais d'extraction étaient du même ordre de grandeur (4,32 f./t.) qu'à Lescalon (4,89 f./t.), mais le rendement en minerai propre à la fusion n'atteignait que $58 \%$ (au licu de $94 \%$ ). Les moyennes pour 12 gites français de ce type s'élevaient respectivement à $1,58 \mathrm{f}$. pour un rendement de $39 \%$.

8. Consulter à ce sujet le travail magistral de J.Y. ANDRIEUX «Forges et hauts foumeaux en Bretagne du XVIF au XIX siècle, cdit. Cid, Nantes, 1987, 328 p. Voir aussi L. ChaURIS. Un projet de haut foumeau à Trégarvan au $\mathrm{XIX}$ siècle, les Cahiers de $1^{\prime} I r o i s e, 37^{\circ}$ annéc, n०3, 1990, p. 156-163.

9. Seuls devaient vraiment se développer, plus tardivement, au $\mathrm{xX}^{*}$ siècle, les gisements de l'Anjou et de Normandic... avant de chuter d'une manière vertigineuse à partir de 1965. Voir L. Chauris. Les exploitations minières dans le Massif armoricain. Déclin ou progrès ? Norois, 141, 1989, p. 5-32.

10. E. DE FourCy, op. cité note 5 . Voir aussi arch. nat. F. 144333 (3).

11. Voir à ce sujet l'article de L. Chauris, op. cité note 8 sur le projet avorté d'un haut fourneau à Trégarvan.

12. L. Chauris. « Impacts sur l'environnement d'une installation industriclle à la campagne à la fin du XVIrr siècle : les forges de Coat-anNoz ", Penn ar Bed, n 134, 1989, p. 4-9. 